USM-TH-82

\title{
From Screening to Confinement in a Gauge-Invariant Formalism
}

\author{
Patricio Gaete *, and Iván Schmidt ${ }^{\dagger}$ \\ Departamento de Física, Universidad Técnica F. Santa Maria, Valparaíso, Chile
}

\begin{abstract}
Features of screening and confinement are reviewed in two-dimensional quantum electrodynamics (QED2). Our discussion is carried out using the gaugeinvariant but path-dependent variables formalism. This alternative and useful approach exploits the rich structure of the electromagnetic cloud or dressing around static fermions in a straightforward and simple way.

PACS number(s): 12.20.Ds, 11.15.Tk
\end{abstract}

\section{INTRODUCTION}

It is well known that one of the long standing problems in physics is understanding confinement of quarks and gluons from first principles. The reason is that infrared divergences and gauge dependence make bound-state equations very difficult to approximate. In this paper we want to address the issue of gauge dependence within the confinement problem. Our purpose is to present a formalism in which everything is expressed in terms of physical (gauge invariant) quantities from the start. As a bonus, the usual qualitative picture of confinement, in terms of an electric flux tube linking quarks [1],2], emerges naturally in this formalism.

\footnotetext{
*E-mail: pgaete@fis.utfsm.cl

$\dagger$ E-mail: ischmidt@fis.utfsm.cl
} 
As a first step we will consider two dimensional gauge theories, which can be regarded as a theoretical laboratory for studying four-dimensional gauge theories. Of particular interest are non-perturbative issues such as confinement and spectrum of models, which can be settled in these theories. Of these, the Schwinger model [3] has probably enjoyed the greatest popularity due to several features that it possesses. For instance, the spectrum contains a massive mode, the charge is screened and confinement is satisfactorily addressed. We also draw attention to the fact that the transition from screening to confinement of probe charges is possible only for nonvanishing spinor mass [4,5].

On the other hand, in recent times a description in terms of gauge-invariant but pathdependent field variables in Abelian gauge theories, and the intimately related question of gauge fixing, has been developed [6, đ]. In particular, it was shown how the gauge fixing procedure corresponds, in this formalism, to a path choice. Therefore this represents a pathdependent but physical QED where a consistent quantization directly in the path space is carried out. Incidentally, it is of interest to notice that the physical electron is not the Lagrangian fermion, which is neither gauge-invariant nor associated with an electric field. Instead, the physical electron is the Lagrangian fermion together with a non-local cloud (or dressing) of gauge fields [8]. This remark opens up the way to a stimulating discussion of how the electromagnetic cloud is distributed around fermions.

Within this framework the aim of the present paper is to reexamine some aspects about screening and confinement in two-dimensional quantum electrodynamics from the viewpoint of the gauge-invariant formalism. This offers a natural setting for such studies, because it involves the use of strings to carry electric flux. Moreover, we obtain computational rules that have clear as well as simple interpretation, in contrast to the standard Wilson loop procedure where subtleties related to the correct calculation must be considered [9, 10]. In Sec. II we present gauge-invariant expressions which will form the basis of our subsequent considerations. Sec. III constitutes the central part of our work. Specifically, we calculate the interaction energy between external probe sources, paying due attention to the structure of the fields that surround the charges. Here we will focus our attention on the transition 
from screening to confinement.

\section{GAUGE-INVARIANT VARIABLES}

Let us start our analysis with a brief presentation of the gauge-invariant variables formalism [6:7]. For this purpose, we introduce the vector gauge-invariant field

$$
\mathcal{A}_{\mu}(y)=A_{\mu}(y)+\partial_{\mu}\left(-\int_{C_{\xi y}} d z^{\mu} A_{\mu}(z)\right),
$$

where the path integral is to be evaluated along some contour $C_{\xi y}$ connecting $\xi$ and $y$. Here $A_{\mu}$ is the usual electromagnetic potential and, in principle, it is taken in an arbitrary gauge. The point we wish to emphasize, however, is that $\mathcal{A}_{\mu}(y)$ is invariant with respect to gauge transformations

$$
A_{\mu}(y) \rightarrow A_{\mu}(y)+\partial_{\mu} \Lambda(y) .
$$

Thus $\mathcal{A}_{\mu}$, while no longer gauge-dependent, now becomes path-dependent. We now choose

the contour as the spacelike straight line $z^{1}=\xi^{1}+\alpha(y-\xi)^{1}$, where $\alpha(0 \leq \alpha \leq 1)$ is the parameter describing the contour and $\xi^{1}$ is an arbitrary (fixed) reference point. Without loss of generality, we can choose $\xi^{1}=0$. This path choice may be made more explicit by splitting Eq.(1) in the form

$$
\begin{aligned}
& \mathcal{A}_{1}(x)=A_{1}(x)-\partial_{1} \int_{0}^{1} d \alpha x^{1} A_{1}\left(\alpha x^{1}\right), \\
& \mathcal{A}_{0}(x)=A_{0}(x)-\partial_{0} \int_{0}^{1} d \alpha x^{1} A_{1}\left(\alpha x^{1}\right),
\end{aligned}
$$

a short calculation yields

$$
\begin{gathered}
\mathcal{A}_{1}\left(x^{0}, x^{1}\right)=0, \\
\mathcal{A}_{0}\left(x^{0}, x^{1}\right)=\int_{0}^{1} d \alpha x^{1} E_{1}\left(\alpha x^{1}\right),
\end{gathered}
$$


where $E_{1}$ is the one-dimensional electric field. The above expressions coincide with the Poincaré gauge conditions [6].

We now turn our attention to the fermion field. In this formalism the charged matter field together with the electromagnetic cloud (dressing) which surrounds it, or what is the same the physical electron, is given by [6,8],

$$
\Psi(y)=\exp \left(-i e \int_{C_{\xi y}} d z^{\mu} A_{\mu}(z)\right) \psi(y) .
$$

Following our path choice, expression ( 7) may be rewritten as

$$
\Psi(y)=\exp \left(-i e \int_{\xi}^{y} d z^{1} A_{1}(z)\right) \psi(y)
$$

It is worthwhile remarking at this point that the breaking of the gauge invariance of the fields in the standard formalism is transformed into breaking of the translational invariance in the path-dependent formalism. The way of solving this problem is letting the reference point $\xi^{1}$ go to infinity.

Before we proceed further, we wish to show that this approach yields interesting results by calculating a gauge-invariant photon propagator. From the expression for the physical fields (1) it can be shown that the gauge-invariant propagator is given by

$$
\begin{array}{r}
\mathcal{D}_{\mu \nu}(x, y)=D_{\mu \nu}(x, y)+\frac{\partial}{\partial x^{\mu}} \int_{x}^{\xi} d z^{\alpha} D_{\alpha \nu}(z, y)+\frac{\partial}{\partial y^{\nu}} \int_{y}^{\xi} d w^{\beta} D_{\mu \beta}(x, w)+ \\
+\frac{\partial}{\partial x^{\mu} \partial y^{\nu}} \int_{x}^{\xi} d z^{\alpha} \int_{y}^{\xi} d w^{\beta} D_{\alpha \beta}(z, w) .
\end{array}
$$

$D_{\mu \nu}(x, y)$ on the right-hand side (RHS) of Eq.(9) is the photon propagator taken in an arbitrary gauge. Implementation of the Poincaré gauge amounts to using the contour $z^{1}=$ $\xi^{1}+\alpha(y-\xi)^{1}$ and $z^{1}=\xi^{1}+\rho(x-\xi)^{1}$. We can choose, for example, $D_{\mu \nu}(x, y)$ in the temporal gauge, that is,

$$
D_{\mu \nu}(x, y)=g_{\mu 1} g_{\nu 1} \delta\left(x^{1}-y^{1}\right)\left(\frac{1}{2}\left|x^{0}-y^{0}\right|+B\left(x^{0}-y^{0}\right)-A\right),
$$

where, as it is well known, the residual gauge invariance manifests itself in the presence of the constants $A$ and $B$. This is a peculiarity of the temporal gauge, which does not fix the gauge uniquely. Then, from Eq.(9), we find that 


$$
\mathcal{D}_{\mu \nu}(x, y)=g_{\mu 0} g_{\nu 0} \frac{1}{2} \delta\left(x^{0}-y^{0}\right)\left(\left|x^{1}-y^{1}\right|-\left|x^{1}-\xi^{1}\right|-\left|y^{1}-\xi^{1}\right|\right) .
$$

Thus one avoids the unphysical features associated with the gauge-dependent formulation. Nevertheless, as it should be expected, the above propagator breaks the translational invariance. The solution to this problem is letting $\xi^{1}$ go to infinity. However, we do not intend to address these problems here. A fuller account on gauge invariant Green's functions will be provided elsewhere [11].

This concludes our brief introduction to gauge invariant variables and gauge conditions.

\section{INTERACTION ENERGY IN QED2}

As already mentioned, our immediate objective is to calculate the interaction energy between external probe sources in the Schwinger model. To do this, we will exploit the rich structure of the electromagnetic cloud or dressing around static fermions.

We shall begin by considering the bosonized form of the Schwinger model [12]:

$$
\mathcal{L}=-\frac{1}{4} F_{\mu \nu}^{2}+\frac{1}{2}\left(\partial_{\mu} \varphi\right)\left(\partial^{\mu} \varphi\right)-\frac{e}{2 \sqrt{\pi}} \varepsilon^{\mu \nu} F_{\mu \nu} \varphi++m \sum(\cos (2 \pi \varphi+\theta)-1)-A_{0} J^{0},
$$

where $J^{0}$ is the external current, $\sum=\frac{e}{2 \pi^{\frac{3}{2}}} \exp \left(\gamma_{E}\right)$ with $\gamma_{E}$ the Euler-Mascheroni constant, and $\theta$ refers to the $\theta$ vacuum.

\section{A. Massless case}

Our purpose is to compute the interaction energy in the $m=0$ case. The first step in this direction is to carry out the integration over $\varphi$ in (12). This allows us to write the effective Lagrangian

$$
\mathcal{L}=-\frac{1}{4} F_{\mu \nu}\left(1+\frac{e^{2}}{\pi} \frac{1}{\partial^{2}}\right) F^{\mu \nu}-A_{0} J^{0} .
$$

It is worthwhile sketching at this point the canonical quantization of this theory from the

Hamiltonian analysis point of view. The canonical momenta are $\Pi^{\mu}=-\left(1+\frac{e^{2}}{\pi} \frac{1}{\partial^{2}}\right) F^{0 \mu}$ with the only nonvanishing canonical Poisson brackets being 


$$
\left\{A_{\mu}(t, x), \Pi^{\nu}(t, y)\right\}=\delta_{\mu}^{\nu} \delta(x-y)
$$

Since $\Pi_{0}$ vanishes we have the usual primary constraint $\Pi_{0}=0$, and $\Pi^{1}=\left(1+\frac{e^{2}}{\pi} \frac{1}{\partial^{2}}\right) F^{10}$. Therefore the canonical Hamiltonian is

$$
H_{C}=\int d x\left(-\frac{1}{2} \Pi_{1}\left(1+\frac{e^{2}}{\pi} \frac{1}{\partial^{2}}\right)^{-1} \Pi^{1}+\Pi^{1} \partial_{1} A_{0}+A_{0} J^{0}\right) .
$$

Requiring the primary constraint $\Pi^{0}=0$ to be preserved in time yields the following secondary constraint

$$
\Omega_{1}(x)=\partial_{1} \Pi^{1}-J^{0} .
$$

It is straightforward to check that there are no more constraints in the theory and that both constraints are first class. The Hamiltonian that generates translations in time is given by

$$
H=H_{C}+\int d x\left(c_{0}(x) \Pi_{0}(x)+c_{1}(x) \Omega_{1}(x)\right)
$$

where $c_{0}(x)$ and $c_{1}(x)$ are arbitrary functions. Furthermore, since $\Pi^{0}=0$ always, and $\dot{A}_{0}(x)=\left[A_{0}(x), H\right]=c_{0}(x)$, we discard $A_{0}(x)$ and $\Pi_{0}(x)$. Therefore the Hamiltonian reduces to

$$
H=\int d x\left\{-\frac{1}{2} \Pi_{1}\left(1+\frac{e^{2}}{\pi} \frac{1}{\partial^{2}}\right)^{-1} \Pi^{1}+c^{\prime}(x)\left(\partial_{1} \Pi^{1}-J^{0}\right)\right\}
$$

where $c^{\prime}(x)=c_{1}(x)-A_{0}(x)$.

According to the usual procedure we introduce a supplementary condition on the vector potential such that the full set of constraints becomes second class, so we write

$$
\Omega_{2}(x)=\int_{0}^{1} d \alpha x^{1} A_{1}(\alpha x)=0
$$

where, as in the previous section, $\alpha$ is the parameter describing a spacelike straight line of integration. It immediately follows that the fundamental Dirac brackets read

$$
\begin{gathered}
\left\{A_{1}(x), A^{1}(y)\right\}^{*}=0=\left\{\Pi_{1}(x), \Pi^{1}(x)\right\}^{*} \\
\left\{A_{1}(x), \Pi^{1}(y)\right\}^{*}=\delta^{(1)}(x-y)-\partial_{1}^{x} \int_{0}^{1} d \alpha x^{1} \delta^{(1)}(\alpha x-y) .
\end{gathered}
$$


It is important to realize that expression (8) represents charged particles together with an associated proper electric field. To see how this arises let $|E\rangle$ be an eigenvector of the electric field operator $E_{1}(x)$, with eigenvalue $\varepsilon_{1}(x)$ :

$$
E_{1}(x)|E\rangle=\varepsilon_{1}(x)|E\rangle .
$$

Next we will consider the state $\Psi(y)|E\rangle$. By means of Eq. (22) we have that

$$
E_{1}(x) \Psi(y)|E\rangle=\Psi(y) E_{1}(x)|E\rangle+\left[E_{1}(x), \Psi(y)\right]|E\rangle
$$

From our above hamiltonian analysis, Eq. (23) may be rewritten as

$$
E_{1}(x) \Psi(y)|E\rangle=\left(\varepsilon_{1}(x)+q\left(1+\frac{e^{2}}{\pi} \frac{1}{\partial_{x}^{2}}\right)^{-1} \int_{0}^{1} d \alpha y_{1} \delta^{(1)}\left(\alpha y_{1}-x_{1}\right)\right) \Psi(y)|E\rangle .
$$

Hence we see that the operator $\Psi(y)$ is the dressing operator of the creation of an electron together with an associated proper electric field. Notice that the integral in Eq.(24) is nonvanishing only on the contour of integration. As a result, we have a static electric field on a line.

At this point we should mention that if we consider a modified form for the electromagnetic cloud in the Poincaré gauge Eq.(8), which is equivalent to the Coulomb gauge [6], that is,

$$
\Psi(y)=\exp \left(-i q \int_{0}^{y} d z^{k} A_{k}^{L}(z)\right) \psi(y)
$$

where $A_{1}^{L}$ refers to the longitudinal part of $A_{1}$, we would obtain that the field $\Psi$ dresses the charge $\psi$ with the electric field :

$$
E_{1}(x) \Psi(y)|E\rangle=\left(\varepsilon_{1}(x)+\frac{q}{2} e^{-\frac{e}{\sqrt{\pi}}\left|x_{1}-y_{1}\right|}\right) \Psi(y)|E\rangle .
$$

In order to calculate the energy between external static charges, we take a fermion localized at $y_{1}^{\prime}$ and an antifermion at $y_{1}$, both dressed according to Eq.(8), and compute the expectation value of the QED2 Hamiltonian in the physical state $|\Omega\rangle$, which we will denote by $\langle H\rangle_{\Omega}$. From our above Hamiltonian structure, we have that 


$$
\langle H\rangle_{\Omega}=\left\langle\Omega\left|\int d x_{1}\left(-\frac{1}{2} \Pi_{1}\left(1+\frac{e^{2}}{\pi} \frac{1}{\partial^{2}}\right)^{-1} \Pi^{1}\right)\right| \Omega\right\rangle .
$$

As mentioned before, the fermions are taken to be static, thus we can substitute $\partial^{2}$ by $-\partial_{1}^{2}$ in Eq.(27). In that case we write

$$
\langle H\rangle_{\Omega}=\left\langle\Omega\left|\int d x_{1}\left(-\frac{1}{2} \Pi_{1}\left(1-\frac{e^{2}}{\pi} \frac{1}{\partial_{1}^{2}}\right)^{-1} \Pi^{1}\right)\right| \Omega\right\rangle .
$$

As has been established by Dirac [8], the physical states $|\Omega\rangle$ correspond to the gauge invariant ones. In this way, the state corresponding to two opposite charges at different points can be made gauge invariant by including a dressing as in Eq.(8), which keeps the entire state gauge invariant. In other words,

$$
|\Omega\rangle \equiv\left|\bar{\Psi}(y) \Psi\left(y^{\prime}\right)\right\rangle=\bar{\psi}(y) \exp \left(-i q \int_{y}^{y^{\prime}} d z^{1} A_{1}(z)\right) \psi(y)|0\rangle,
$$

where $|0\rangle$ is the physical vacuum state.

We are now ready to calculate $\langle H\rangle_{\Omega}$. Using our formalism, we can show that

$$
E_{1}(x)|\Omega\rangle=\bar{\Psi}(y) \Psi\left(y^{\prime}\right) E_{1}(x)|0\rangle+q\left(1-\frac{e^{2}}{\pi} \frac{1}{\partial_{1}^{2}}\right)^{-1} \int_{y}^{y^{\prime}} d z_{1} \delta\left(x_{1}-z_{1}\right)|\Omega\rangle .
$$

Inserting this into Eq. (28), the energy in the presence of the static charges will be given by

$$
\langle H\rangle_{\Omega}=\langle H\rangle_{0}+\frac{q^{2}}{2} \frac{\sqrt{\pi}}{e}\left(1-e^{-\frac{e}{\sqrt{\pi}}\left|y-y^{\prime}\right|}\right),
$$

where $\langle H\rangle_{o}=\langle 0|H| 0\rangle$. Since the potential is given by the term of the energy which depends on the separation of the two fermions, from the expression (31) we obtain

$$
V=\frac{q^{2}}{2} \frac{\sqrt{\pi}}{e}\left(1-e^{-\frac{e}{\sqrt{\pi}}\left|y-y^{\prime}\right|}\right)
$$

Thus we have demonstrated that the potential between fermions can be directly obtained once the structure of the photonic clouds around static fermions is known. In this case expression (32) is the expected screening contribution to the potential. Physically this means that the initial string was broken and all the charges are screened. In other terms, as a result of the interaction with massless fermions the original Coulomb potential ( proportional to 
the distance ) is screened. This feature is similar to that expected for QCD strings in the adjoint representation.

Before we conclude this subsection, it is important to notice that with the path choice stated in Eq.(25) (modified Poincaré gauge), and from the previous canonical formalism, we can write a scalar potential

$$
\mathcal{A}_{o}(t, x)=\int_{0}^{1} d \alpha x^{1} E_{1}^{L}(t, \alpha x)=\int_{0}^{1} d \alpha x^{1}\left(1-\frac{e^{2}}{\pi} \frac{1}{\partial_{1}^{2}}\right)_{\alpha x}^{-1} \frac{\partial_{1}^{\alpha x}\left(-J^{0}(\alpha x)\right)}{\partial_{\alpha x}^{2}},
$$

where the superscript $L$ refers to the longitudinal part and $J^{0}$ is the external source. Accordingly, the potential for a pair of static pointlike opposite charges located at $y$ and $y^{\prime}$, that is, $J^{0}(t, x)=q\left\{\delta(x-y)-\delta\left(x-y^{\prime}\right)\right\}$, is given by

$$
V=q\left(\mathcal{A}_{0}(y)-\mathcal{A}_{0}\left(y^{\prime}\right)\right)=\frac{q^{2}}{2} \frac{\sqrt{\pi}}{e}\left(1-e^{-\frac{e}{\sqrt{\pi}}\left|y-y^{\prime}\right|}\right) .
$$

It is gratifying to notice here the simplicity and directness of this derivation, which is manifestly gauge-invariant.

\section{B. Massive case}

We now proceed to consider the massive case. For this purpose we have to carry out the integration over $\varphi$ in (12). But since this expression is non-polynomial in $\varphi$, we expand the effective Lagrangian in terms of $F^{\mu \nu}$. Thus it follows that

$$
\mathcal{L}=-\frac{1}{4} F_{\mu \nu}^{2}-\frac{e^{2}}{4 \pi} F_{\mu \nu} \frac{1}{\partial^{2}+4 \pi m \Sigma} F^{\mu \nu}-A_{0} J^{0}
$$

where we have taken $\theta=0$. If we now look at the limit of slowly varying fields, we find that

$$
\mathcal{L}=-\frac{1}{4} F_{\mu \nu}\left(1+\frac{e^{2}}{4 \pi^{2} m \Sigma}\right) F^{\mu \nu}-A_{0} J^{0}
$$

As in the previous subsection, our objective will be to calculate the potential energy for this theory. However, as we now know, this calculation is facilitated by using the expression:

$$
\mathcal{A}_{0}(t, x)=\int_{0}^{1} d \alpha x^{1} E_{1}^{L}(t, \alpha x)
$$


Thus we obtain

$$
\mathcal{A}_{0}(t, x)=\left(1+\frac{e^{2}}{4 \pi^{2} m \Sigma}\right)^{-1} \int_{0}^{1} d \alpha x^{1} \frac{\partial_{1}^{\alpha x}\left(-J^{0}(\alpha x)\right)}{\partial_{\alpha x}^{2}} .
$$

For $J^{0}(t, \alpha x)=q \delta(\alpha(x-a))$ expression (38) then becomes

$$
\mathcal{A}_{0}(t, x)=\frac{q}{2}\left(1+\frac{e^{2}}{4 \pi^{2} m \Sigma}\right)^{-1}|x-a| .
$$

By means of expression (39) we evaluate the interaction energy for a pair of static pointlike opposite charges at $y$ and $y \prime$, as

$$
V=q\left(\mathcal{A}_{0}(y)-\mathcal{A}_{0}(y \prime)\right)=\frac{q^{2}}{2}\left(1+\frac{e^{2}}{4 \pi^{2} m \Sigma}\right)^{-1}\left|y-y^{\prime}\right| .
$$

Considering the limit $m \ll e$, we get

$$
V=\frac{q^{2}}{e^{2}} 2 \pi^{2} m \Sigma\left|y-y^{\prime}\right|
$$

This can be recognized as the standard result for the interaction potential [4], which is also just the confinement contribution to the potential. This derivation tells us that one can in fact interpolate between screening and confinement as soon as the dynamical fermions have a nonvanishing mass. It is therefore of interest to reexamine the transition between these limits. To see how this arises in this formalism, we start with Eq.(35). Using the fact that the fields are taken to be static, which means substituting $\partial^{2}$ by $-\partial_{1}^{2}$, we get

$$
\mathcal{L}=-\frac{1}{4} F_{\mu \nu}^{2}+\frac{e^{2}}{4 \pi} F_{\mu \nu} \frac{1}{\partial_{1}^{2}-4 \pi m \Sigma} F^{\mu \nu}-A_{0} J^{0}
$$

Thus, in the present approach, the scalar potential (37) may be written as

$$
\mathcal{A}_{0}(t, x)=\int_{0}^{1} d \alpha x^{1} \partial_{1}^{\alpha x}\left(-\frac{J^{0}(\alpha x)}{\partial_{\alpha x}^{2}-\lambda^{2}}\right)-4 \pi m \Sigma \int_{0}^{1} d \alpha x^{1} \frac{\partial_{1}^{\alpha x}\left(-J^{0}(\alpha x)\right)}{\left(\partial^{2}-\lambda^{2}\right)_{\alpha x} \partial_{\alpha x}^{2}},
$$

where $\lambda^{2} \equiv \frac{e^{2}}{\pi}+4 \pi m \Sigma$. For $J^{0}(t, \alpha x)=q \delta(\alpha(x-a))$ Eq. 43) reduces to

$$
\mathcal{A}_{0}(t, x)=\frac{q}{2 \lambda}\left(1+\frac{4 \pi m \Sigma}{\lambda^{2}}\right)\left(1-e^{-\lambda a}\right)+\frac{q}{2}\left(1-\frac{\frac{e^{2}}{\pi}}{\lambda^{2}}\right) a .
$$

Expression (44) immediately shows that the potential for two opposite charges located at $y$ and $y \prime$ is given by 


$$
V=\frac{q^{2}}{2 \lambda}\left(1+\frac{4 \pi m \Sigma}{\lambda^{2}}\right)\left(1-e^{-\lambda|y-y \prime|}\right)+\frac{q^{2}}{2}\left(1-\frac{\frac{e^{2}}{\pi}}{\lambda^{2}}\right)|y-y \prime| .
$$

It is straightforward to check that in the limit $m=0$, expression (45) reduces to (34).

Until now we have taken the vacuum angle $\theta$ as zero. We now want to consider the nonvanishing $\theta$ contribution to the potential that would follow from this formalism. With this in mind, we start by writing

$$
\mathcal{L}=-\frac{1}{4} F_{\mu \nu} F^{\mu \nu}-\frac{e^{2}}{4 \pi} F_{\mu \nu} \frac{1}{\partial^{2}+4 \pi m \Sigma} F^{\mu \nu}+e m \Sigma \frac{1}{\partial^{2}+4 \pi m \Sigma} \theta \varepsilon_{\mu \nu} F^{\mu \nu}-A_{0} J^{0} .
$$

Since we are dealing with static fermions, we can substitute $\partial^{2}$ by $-\partial_{1}^{2}$. In that case we write

$$
\mathcal{L}=-\frac{1}{4} F_{\mu \nu}\left(1-\frac{e^{2}}{\pi} \frac{1}{\partial_{1}^{2}-4 \pi m \Sigma}\right) F^{\mu \nu}-e m \Sigma \frac{1}{\partial_{1}^{2}-4 \pi m \Sigma} \theta-A_{0} J^{0}
$$

But since the first term on the right-hand side (RHS) of Eq. (47) gave as a result the potential given in Eq. (42), we only need to consider the second, $\theta$ dependent, term. Using (37), we may write the expression for the scalar potential in the form

$$
\mathcal{A}_{0}^{\theta}(t, x)=\int_{0}^{1} d \alpha x^{1} 2 e m \Sigma\left(\partial_{1}^{2}-\lambda^{2}\right)^{-1} \theta
$$

where $\lambda^{2} \equiv \frac{e^{2}}{\pi}+4 \pi m \Sigma$. If we now look at the limit of slowly varying fields, we find that

$$
\mathcal{A}_{0}^{\theta}(t, x)=-\int_{0}^{1} d \alpha \frac{2 e m \Sigma}{\lambda^{2}} x^{1} \theta=-\frac{e}{2 \pi}\left(1-\frac{\frac{e^{2}}{\pi}}{\lambda^{2}}\right) x^{1} \theta .
$$

Hence we see that the potential for two opposite charges ( for $q=e$ ) at $y$ and $y^{\prime}$ is given by

$$
V^{\theta}=-\frac{e^{2}}{2}\left(1-\frac{\frac{e^{2}}{\pi}}{\lambda^{2}}\right) \frac{\theta}{\pi}\left|y-y^{\prime}\right|
$$

It is also, up to the $-\frac{\theta}{\pi}$ factor, just the Coulomb potential. From this expression we see that, in the limit of slowly varying fields, the inclusion of the $\theta$ term would reduce the potential to

$$
V=\frac{e^{2}}{2}\left(1-\frac{\frac{e^{2}}{\pi}}{\lambda^{2}}\right)\left(1-\frac{\theta}{\pi}\right)\left|y-y^{\prime}\right|
$$

An immediate consequence of this is that for $\theta=\pi$ the confinement term vanishes [5]. 


\section{ACKNOWLEDGMENTS}

P.G. would like to thank J. Gamboa for the discussions. 


\section{REFERENCES}

[1] T. Muta, Foundations of Quantum Chromodynamics (World Scientific, Singapore, 1987).

[2] T. P. Cheng and L. F. Li, Gauge Theory of Elementary Particle Physics (Clarendon Press, Oxford, 1984).

[3] J. Schwinger, Phys. Rev. 128, 2425 (1962).

[4] D. J. Gross, I. R. Klebanov, A. V. Matytsin and A. V. Smilga, Nucl. Phys. B461, 109 (1996).

[5] E. Abdalla, R. Mohayaee and A. Zadra, Int. J. Mod. Phys. A12, 4539 (1997).

[6] P. Gaete, Z. Phys. C76, 355 (1997).

[7] P. Gaete, Phys. Rev. D59, 127702 (1999).

[8] P. A. M. Dirac, The Principles of Quantum Mechanics (Oxford University Press, Oxford,1958),4th ed.; Can. J. Phys. 33, 650 (1955).

[9] P. E. Haagensen and K. Johnson, eprint hep-th/9702204.

[10] H. A. Falomir, R. E. Gamboa Saravi and F. A. Schaposnik, Phys. Rev. D25, 547 (1982).

[11] P. Gaete and I. Schmidt, " Gauge-Invariant Green's Functions in Abelian Gauge Theories", in preparation.

[12] S. Coleman, R. Jackiw, and L. Susskind, Ann. Phys. (N.Y.) 93, 267 (1975). 